\title{
35 Die Krim nach dem Zweiten Weltkrieg
}

\begin{abstract}
Medea Mendez, geborene Sinopli, war, abgesehen von ihrer Ende der zwanziger Jahre nach Moskau verzogenen jüngeren Schwester Alexandra, die letzte reinrassige Griechin in ihrer Familie, die sich vor Urzeiten an den mit Hellas verwandten taurischen Gestaden angesiedelt hatte. Sie war auch die letzte in der Familie, die noch eine Art Griechisch sprach, vom Neugriechischen ebenso tausend Jahre entfernt wie das Altgriechische von der nur in den taurischen Kolonien erhaltenen mittelalterlichen pontischen Mundart. Unterhalten konnte sie sich seit langem mit niemandem mehr in dieser abgetragenen klangvollen Sprache [...]. Die taurischen Griechen in Medeas Alter waren entweder ausgestorben oder ausgesiedelt worden, sie aber war auf der Krim geblieben, was sie, wie sie meinte, Gottes Gnade verdankte, zum Teil aber auch ihrem spanischen Witwennamen, ihr verblieben von ihrem verstorbenen Mann, einem fröhlichen jüdischen Dentisten mit kleinen, aber auffälligen Fehlern und großen, aber tief verborgenen Vorzügen. ${ }^{1}$
\end{abstract}

Der 1996 im russischen Original erschienene Roman „Medea und ihre Kinder. Eine Familienchronik“ („Medeja i ee deti. Semejnaja Chronika“) stammt von der mittlerweile zwischen Russland und Israel pendelnden Schriftstellerin und PutinGegnerin Ljudmila Ulickaja. Er erzählt das Leben einer weitverzweigten sowjetischen, d.h. multinationalen Familie, die zwischen den Zentren der UdSSR und einer ihrer Peripherien, der Halbinsel Krim nämlich, verstreut lebt. Deren auch emotionalen Mittelpunkt bildet mit der Titelheldin Medea eine Nachfahrin pontischer GriechInnen. Dem Schicksal der übrigen KrimgriechInnen nach dem Zweiten Weltkrieg, die wie die TatarInnen oder BulgarInnen der Halbinsel in ihrer Mehrheit vom stalinistischen Regime deportiert worden waren, soll sie, so ihre Erfinderin Ulickaja, wegen ihrer Ehe mit einem sowjetischen Juden entgangen sein. Die jüdische Bevölkerung galt in der Sowjetunion bekanntlich als eigenständige Nationalität, und die Romanfigur Medea lebte somit in einer gemischtnationalen Ehe, die sie vor der Verbannung schützte. Der Vorname der Protagonistin - Medea - führt uns abermals in die klassische Antike und zu der mit dieser Region verbundenen Mythenwelt: Medea war die Tochter des Königs Aietes von Kolchis, an der Ostküste des Schwarzen Meeres, im Gebiet des heutigen Georgiens gelegen. Die Argonauten unter der Führung Iasons erbeuten, so will es der Mythos, mit Hilfe der in Liebe zu Iason entbrannten Medea das von ihrem Vater gehütete Goldene Vlies. Medea und Iason müssen deshalb aus Kolchis fliehen, heiraten und bekommen miteinander zwei Söhne, die sie tötet, nachdem Iason sich der Tochter Kreons zugewandt und sie verstoßen hat.

1 Ulitzkaja (1997), 5 f. In der deutschen Übersetzung fehlt der im russischen Original beigegebene Untertitel.

Ә OpenAccess. (c) 2020 Kerstin S. Jobst, publiziert von De Gruyter. (cc))BY Dieses Werk ist lizenziert unter der Creative Commons Attribution 4.0 International. https://doi.org/10.1515/9783110520620-037 
Das Medea-Thema ist ein in der Kunst vielfach verarbeitetes Sujet, das u.a. Euripides, Seneca (ca. 1-65 n.Chr.), Franz Grillparzer (1791-1872), Hans Henny Jahn (1894-1959) oder Christa Wolf (1929-2011) in ganz unterschiedlichen Formen aufgegriffen haben. Ulickajas Medea aber hat mit ihrer mythischen Namensvetterin nur wenig gemein, für den Slavisten Wolfgang Kasack ist sie vielmehr vor allen Dingen ein „Kraftfeld, auf das eine unüberschaubare Menge von Verwandten ausgerichtet ist“, die sich aus „Menschen vieler Nationen“ zusammensetzt. Medea symbolisiert für ihn „das Verbundensein der Menschen überhaupt.“2 Sie versinnbildlicht aber zugleich die keinesfalls nur von den sowjetischen Führern propagierte, sondern in Ansätzen existente sowjetische Völkerfamilie: Die Menschen heirateten oder verbanden sich auf sonstige Weise miteinander über die ethnischen Grenzen hinweg und kommunizierten in der lingua franca der UdSSR, dem Russischen. Der Roman spiegelt das Schicksal einer Handvoll familiär verbandelter Menschen im 20. Jahrhundert wider, ohne die Brüche und Zäsuren - die Revolution, den Aufbau des Sozialismus, den Zweiten Weltkrieg, die Deportationen - in den Mittelpunkt der Erzählung zu rücken; gleichwohl werden diese Ereignisse in ihrem Einfluss auf die Figuren spürbar. Deutlich wird deren Beziehung nicht nur zu Medea, sondern auch zur Halbinsel selbst: Man spürt deren Freude, an diesem schönen Ort ihre Ferien verbringen zu können, selbst wenn dessen Polyethnizität in der zweiten Hälfte des 20. Jahrhunderts nicht mehr vorhanden war, sieht man von ,Relikten“ wie der griechischstämmigen Medea einmal ab.

Die Krim war nach dem Zweiten Weltkrieg eine andere geworden, was nicht nur an den weitläufigen Zerstörungen lag, sondern eben auch an dem Menschenverlust durch Kampfhandlungen, Massaker und Deportationen, welche in heutiger Terminologie als Genozid (so die Shoa) und ethnische Säuberungen zu bezeichnen sind, denen ebenfalls genozidale Elemente innewohnten. Weiteres kam hinzu: Es änderte sich der Status der Halbinsel innerhalb des staatlichen Gefüges, wurde doch ein gutes Jahr nach der Deportation der tatarischen Bevölkerung, am 30. Juni 1945 die seit Anfang der 1920er Jahre existierende Autonome Sozialistische Sowjetrepublik aufgelöst. Die Halbinsel war für die nächsten Jahre eine sog. oblast' (d.h. ein Verwaltungsbezirk) innerhalb der Russländischen Sozialistischen Föderativen Sowjetrepublik (RSFSR). Öffentlich bekanntgemacht wurde dieser Statuswechsel allerdings erst ein Jahr später, nämlich Ende Juni 1946. Als Grund dafür und für die Umsiedlung der TatarInnen wurde abermals die vermeintliche Massenkollaboration mit den Nationalsozialisten angeführt. Und

2 Kasack (1997), 732. 
wieder wurde der Anteil tatarischer Menschen an der Befreiung von den Invasoren als Rotarmisten oder Partisanen verschwiegen. ${ }^{3}$

Bezeichnend war ebenso, dass die sowjetische Führung und Administration vor Ort danach strebten, die Erinnerung an die deportierten Völker, allen voran das krimtatarische, auszulöschen: Die während der korenizacija (Kapitel 32) in den 1920er Jahren gedruckten Schulbücher in krimtatarische Sprache wurden verbrannt, Moscheen, Friedhöfe etc. zerstört, topographische Bezeichnungen krimtatarischer (zuweilen auch vortatarischer) Provenienz umgewidmet. Diese Politik der Ausmerzung älterer, nun missliebiger historischer Spuren ist nicht neu: Während unter Katharina II. noch gräzisierten Namensformen der Vorzug gegenüber tatarischen Toponymen gegeben worden war (Kapitel 23), war nun Russifizierung das Stichwort; so wurde beispielsweise aus Qarasuvbazar „Belogorsk“ oder aus Bağçasaray „Puškinskij“, was zugleich eine Reminiszenz an Alexander Puškin und sein Poem „Bachčisarajskij fontan“ war (Kapitel 2). Diese kulturelle und administrative Russifizierung, wie Brian G. Williams dieses Vorgehen bezeichnet hat, ${ }^{4}$ wurde begleitet von einer zielgerichteten Einwanderung russischer und ukrainischer SiedlerInnen. Diese sollten die entvölkerten ländlichen Regionen wiederbeleben und die dringend gebrauchten Arbeitskräfte stellen. Letztlich erscheint dieses Vorgehen als Wiederholung der Politik, welche das zarische Russland knapp hundert Jahre vordem, also nach dem Krimkrieg, verfolgt hat, als es die ausgereiste tatarische Bevölkerung ebenfalls durch Slaven ersetzte (vgl. Kapitel 28). Es wird geschätzt, dass ca. neunzig Prozent der slavischen Krim-Bevölkerung erst nach dem Zweiten Weltkrieg auf die Halbinsel gelangt sei. ${ }^{5}$ Erst seitdem war diese im wahrsten Wortsinn ,russisch geworden'.

Die Weltöffentlichkeit nahm die Krim aber nicht unbedingt wegen der auf ihr stattfindenden ethnopolitischen Verwerfungen wahr, sondern vielmehr wegen der Konferenz von Jalta, die vom 4. bis zum 11. Februar 1945 dort abgehalten wurde. Bei diesem diplomatischen Treffen der alliierten Staatschefs der USA (Franklin D. Roosevelt, 1882-1945), Großbritanniens (Winston Churchill, 18741965) und der Sowjetunion (Josef Stalin) im Livadija-Palast bei Jalta ging es vor allen Dingen um die Zukunft des sich damals bereits in Agonie befindenden Deutschen Reichs, das ein Vierteljahr später endlich kapitulieren sollte. Weitere Themen in Jalta waren die europäische Nachkriegsordnung, die Einbeziehung Frankreichs in den Kreis der Siegermächte sowie der Eintritt der Sowjetunion in den Krieg gegen Japan, der nach der Kapitulation Deutschlands geschehen sollte.

3 Vgl. u. a. Fisher (1978), $167 \mathrm{f}$.

4 Williams (2001), 404.

5 Guboglo u. Chervonnaia (1995), 39. 
Die in Jalta erfolgte Festlegung der sog. Einflusssphären in Europa zwischen den Alliierten machte diese Konferenz, so hat es Stefan Troebst plausibel dargelegt, allein für Polen zu einem (allerdings negativ besetzten) Erinnerungsort. Während zum Beispiel im deutschen Kollektivgedächtnis die Potsdamer Konferenz (August 1945) diejenige der sog. Dreimächtekonferenzen ${ }^{6}$ war, die sich am nachhaltigsten eingeprägt hat, und mit dem Begriff „Jalta“ nur wenig verbunden wird, ist „Jalta“ in polnischen Ohren eine Chiffre für eine ungerechte Nachkriegsordnung. Diese habe Polen - immerhin ein aktiver alliierter Verbündeter vom Exil aus - der Sowjetunion ausgeliefert. ${ }^{7}$ Worauf fußte diese Sichtweise? Die im Zuge des Geheimen Zusatzprotokolls im Hitler-Stalin-Pakt von 1939 an die UdSSR gelangten östlichen Territorien der Zweiten Polnischen Republik verblieben nach den Beschlüssen von Jalta bei der Sowjetunion. Als Kompensation erhielt das neue Polen Teile Ostpreußens und bis dahin deutsche Gebiete östlich der Flüsse Oder und Neiße. Die bürgerliche polnische Exilregierung wurde durch eine prosowjetische ersetzt und die Umsiedlung weiter Bevölkerungsteile beschlossen. Während die Aussiedlung der deutschen Bevölkerung aus den neuen polnischen Gebieten im polnischen Diskurs weniger umstritten war, wurde die in Jalta nicht offen kommunizierte Vertreibung eingewurzelter Polen und Polinnen aus den nun der UdSSR zugeschlagenen Gebieten naturgemäß beklagt. So oder so: Jalta - und damit eben auch die Krim - steht somit für Teile der polnischen Bevölkerung für einen Verrat des Westens am Vaterland. Neben dem (positiven) Sarmatenmythos (Kapitel 2) wird mit der Halbinsel also auch ein negativer Mythos verbunden.

Das nächste Ereignis, welches der Krim über das eigentliche Territorium hinaus eine gewisse Aufmerksamkeit hätte bescheren können, aber aufgrund der sowjetischen Propagandastrategie weithin unbeachtet blieb, fand 1954 statt, also ein Jahr nach dem Tod Stalins. Gemeint ist die häufig, aber unzutreffend als sog. Chruščevsche Schenkung bezeichnete Übergabe der oblast' Krim aus dem Territorialbestand der RSFSR in den der Ukrainischen Sowjetrepublik. Die Öffentlichkeit nahm diesen Transfer vor dem Hintergrund umfänglicher Feierlichkeiten anlässlich des dreihundertsten Jahrestags der Übereinkunft von Perejaslav 1654 wahr; damals hatten sich bekanntlich die ukrainischen Kosaken unter Chmel'nyc'kyj dem Zaren Alexej Michajlovič unterstellt. Dies wird in der ukrainischen Historiographie lediglich als temporäre, pragmatische Verbindung gegen den polnischen König gesehen. Die russische Geschichtsschreibung interpretiert dieses Vertragswerk lieber mit Rekurs auf den ersten ostslavischen Staat der Kiewer

6 Die erste Dreimächtekonferenz hatte Ende 1943 in Teheran stattgefunden.

7 Ich folge hier Troebst (2017), $344 \mathrm{f}$. 


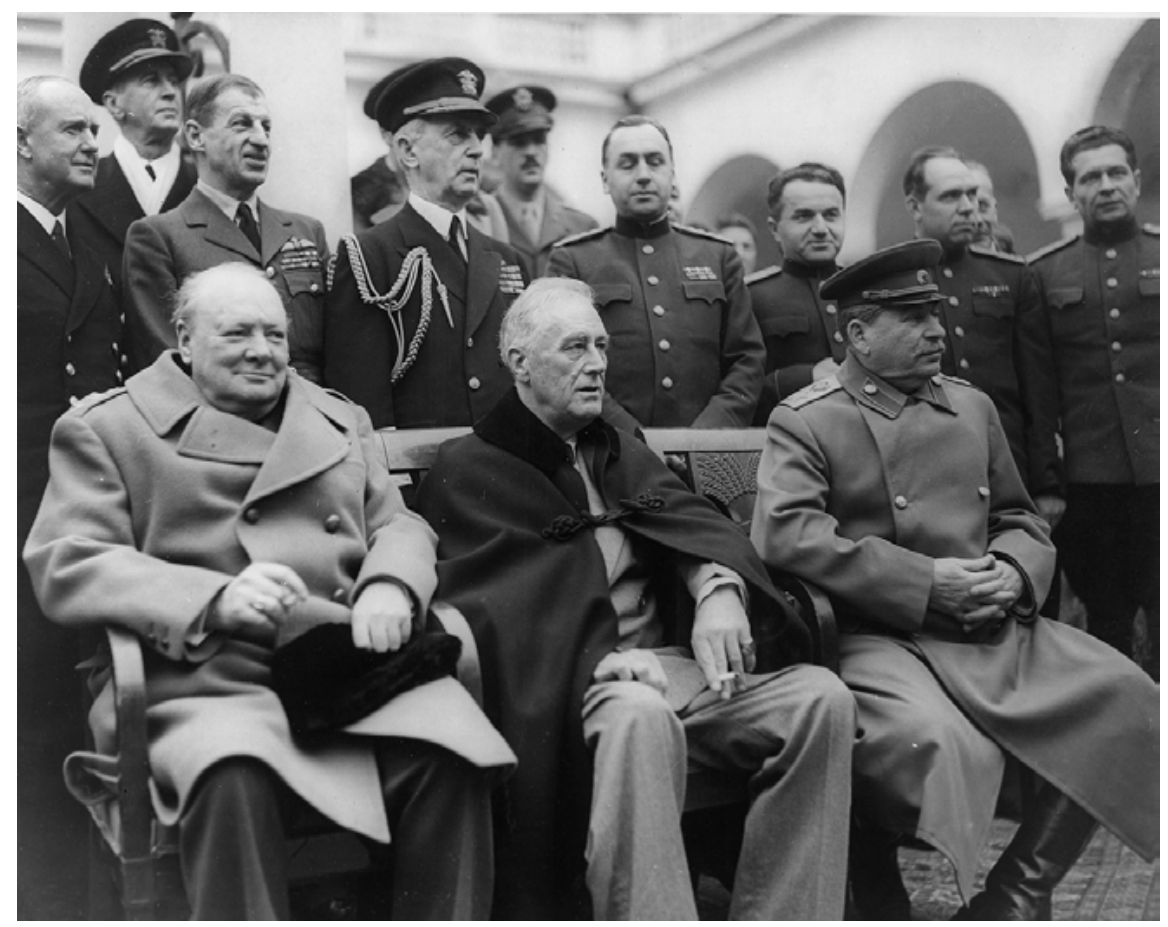

Abb. 13: Gruppenfoto bei der Konferenz von Jalta, 1945 (von links: Winston Churchill, Franklin D. Roosevelt und Josef Stalin)

Rus' als eine Wiedervereinigung zwischen ,Groß-' und ,Kleinrussen' (= Ukrainer). ${ }^{8}$ In jedem Fall wurde 1954 der Transfer der Krim als eine Art Unterpfand für die ,unverbrüchliche Freundschaft` zwischen diesen beiden zahlenmäßig größten Nationalitäten der Union präsentiert.

Die politischen Hintergründe der Feiern der Ereignisse von 1654 und die Unterstellung der Krim unter die Jurisdiktion der Ukraine sind komplex und lassen sich - dies sei vorangestellt - bis heute nicht abschließend klären. Und offenbar wurde dies nicht einmal versucht, wie die Politikwissenschaftlerin Gwendolyn Sasse bereits vor Jahren feststellen musste. Sie präsentiert ihrerseits einige verschiedene Varianten und mögliche Erklärungen, die ein wenig Licht in

8 Plokhy (2017), 282, weist zu Recht auf die unterschiedlichen Bewertungen durch die sowjetische Geschichtsschreibung hin: Während zu Beginn der 1920er Jahre diese Übereinkunft noch als das Zarentum stärkend und damit negativ beurteilt wurde, geriet man später zu positiveren Einschätzungen des Russländischen Imperiums. 
die Angelegenheit bringen. ${ }^{9}$ Folgendes steht außer Zweifel: Von einem Alleingang Chruščevs konnte in dieser Angelegenheit nicht die Rede sein, so dass sich der Begriff ,Schenkung، schon aus diesem Grund verbietet. Wohl wegen seiner engen persönlichen Beziehungen zur Ukraine wurde häufig argumentiert, Chruščev habe der Ukraine mit der Krim eine besondere Ehre erweisen wollen oder - weniger elegant ausgedrückt - die Halbinsel „wie ein[en] Sack Kartoffeln“ (Vladimir Putin) weggegeben. ${ }^{10}$ Die politische Gemengelage im Kreml und der noch nicht abgeschlossene Machtkampf um die Nachfolge Stalins stehen dieser Interpretation allerdings entgegen, verfügte Chruščev doch zu diesem Zeitpunkt noch nicht über den notwendigen Einfluss. Zumindest das Präsidium des Zentralkomitees der KPdSU unter Georgij M. Malenkov (1901-1988) habe diese Entscheidung mittragen müssen, so vermutet der ukrainische Historiker Jurij Šapoval. ${ }^{11}$ Debattiert wurde auch darüber, ob mit der Übergabe an die Ukraine dieser nicht ein erheblicher Teil der logistischen und finanziellen Lasten für den Wiederaufbau der durch den Krieg zerstörten Krim aufgebürdet werden sollte. Einer gewissen Plausibilität entbehrt auch folgende Erklärung nicht, nach der die Ukraine somit ihren Teil der Verantwortlichkeit für die moralisch und völkerrechtlich verwerfliche Massenumsiedlung eingewurzelter Nationalitäten von der Krim übernehmen musste. ${ }^{12}$ Auch ganz pragmatische Überlegungen könnten eine Möglichkeit darstellen, da durch die (bis zum Sommer 2017) fehlende Landverbindung zwischen der Halbinsel und der RSFSR die Versorgung und der Wiederaufbau von der Ukraine aus leichter zu bewerkstelligen waren. ${ }^{13}$ Vermutlich spielte also, wie so oft in der Geschichte, eine ganze Reihe von Gründen eine Rolle.

In jedem Fall sollte die Eingliederung der Krim in die Ukrainische Sowjetrepublik auch im Zusammenhang mit dem russisch-ukrainischen Verhältnis und im gesamtsowjetischen Kontext betrachtet werden. Die Krim war ohne Zweifel ein „verschwenderisches Geschenk“ (,lavish gift“) Moskaus an Kiew, so die Feststellung Plokhys, ${ }^{14}$ und sollte die ,Wiedervereinigung“ Russlands und der Ukraine auf der symbolischen Ebene stärken. Dies gilt übrigens genauso für eine andere Maßnahme: der bereits bei der Gründung der Vereinten Nationen sowohl der Ukraine als auch der Weißrussischen SSR zuerkannte eigene Sitz in der dortigen Vollversammlung, was nur mit Billigung der Westalliierten geschehen konnte. Die

9 Ich halte mich im Folgenden an Sasse (2007), besonders 107-128, sowie an Plokhy (2017), 280284.

10 Vgl. Hromenko (2017), 198-203.

11 Šapoval (2009).

12 Potichnyj (1975), hier 308.

13 So z. B. Subtelny (2000), 500.

14 Plokhy (2017), 283. 
zweitgrößte ostslavische Nationalität wurde nach dem Zweiten Weltkrieg zur secunda inter pares nach der russischen. Dies wurde von den nicht-slavischen Nationalitäten als Botschaft verstanden, denn ungeachtet aller Beschwörungen des Sowjetpatriotismus dominierte politisch und demographisch eben doch das slavische Element in der UdSSR. Die mit der ,Schenkung“ verbundenen Signale an die Ukrainische SSR und ihre Nomenklatura waren ebenfalls komplex: Chruščev konnte sich der besonderen Unterstützung der ukrainischen politischen Elite zwar relativ sicher sein, aber die Krim als zusätzliche Morgengabe Moskaus schadete nicht. Zugleich wurde mit der Halbinsel ein Territorium Teil der Ukraine, dessen Bevölkerung ganz überwiegend russisch und in einem noch höheren Maße russophon war, verwendeten (und verwenden) doch die meisten ethnischen UkrainerInnen auf der Krim Russisch als Erstsprache. In Anbetracht des Umstandes, dass der Kreml bereits in den 1920er Jahren Angst vor sog. ukrainischen Nationalbolschewisten gehabt hatte, ${ }^{15}$ mag die Stärkung des russischen Bevölkerungsteils in dieser Sowjetrepublik ein erwünschter Nebeneffekt gewesen sein. Plausibel ist aber vor allen Dingen eines: Zum Zeitpunkt der ,Schenkung' stand die Sowjetunion nach dem gewonnenen Weltkrieg und dem Aufstieg zur Superund Atommacht auf dem Zenit ihrer Macht. Der Zerfall dieses sozialistischen Imperiums erschien allen als undenkbar. Und in Anbetracht der trotz föderaler Bekenntnisse stark zentralistischen Staatsstruktur war die Zugehörigkeit eines bestimmten Territoriums zu der einen oder anderen Sowjetrepublik nur von eingeschränkter praktischer Relevanz. Oder anders gesagt: Dass die schöne Krim für Russinnen und Russen jemals Ausland werden würde, war weder 1954 noch in den Dekaden danach denkbar. 1991 freilich wurden die Karten neu gemischt (Kapitel 36).

Auch wenn eine befriedigende Untersuchung der politischen und ökonomischen Entwicklung der Krim nach dem Zweiten Weltkrieg und der Eingliederung in die Ukrainische SSR bislang fehlt, ${ }^{16}$ so können doch folgende Aussagen getroffen werden: Weiterhin war die Entwicklung der Halbinsel von der gesamtstaatlichen abhängig, wobei lokale Handlungsoptionen, die selbst die Sowjetunion bot, genutzt wurden. Die Situation war demnach ambivalent: Wie schon 1921/22 und 1932/33, so war auch die Krim von der durch Dürre und Zerstörung hervorgerufenen Hungersnot 1946/47 betroffen, allerdings fielen dieser durch den

15 Vgl. z. B. Jobst (2015b), 199-201.

16 Für Sevastopol' nach 1944 gilt dies nicht, da Qualls (2009) diese Lücke weitgehend geschlossen hat. Bislang unerschlossenes Quellenmaterial zur Moskauer Wirtschaftspolitik gegenüber der Krim im Zusammenspiel mit Kiew hat Sasse (2007), besonders 121-126, konzis bearbeitet und bewertet. 
Zugang $\mathrm{zu}$ frischem Fisch vergleichsweise weniger Menschen zum Opfer. ${ }^{17}$ Die Krim war, wie andere von der sowjetischen Armee rückeroberte Gebiete, großflächig zerstört worden. Städtische Gebiete waren besonders umkämpft gewesen - so Sevastopol' (neben Kerč’ im Osten der Halbinsel), wo im Jahr 1944 nur drei Prozent der Bausubstanz aus der Vorkriegszeit noch intakt war. Dazu beigetragen hatte die weithin praktizierte Taktik der deutschen Wehrmacht, beim Rückzug möglichst große Schäden im zu räumenden Gebiet zu hinterlassen. Im Fall der Krim war u.a. die Wasserversorgung zerstört worden. ${ }^{18}$ Da die Halbinsel seit jeher unter Wassermangel litt, wog dies besonders schwer und führte in der unmittelbaren Nachkriegszeit zu Epidemien. Projekte wie der 1961 begonnene Bau des Nord-Krim-Kanals, der vor allen Dingen die nordöstlichen Gebiete mit Wasser versorgte, ${ }^{19}$ konnten längerfristig Entlastung bringen. Neben dem Wiederaufbau zerstörter Gebäude und Infrastruktur gehörte die Repeuplierung der Halbinsel zu den gravierenden Problemen. Der Bevölkerungs- und damit eben auch Arbeitskräftemangel rührte nicht nur von der Besatzungspolitik, der Shoa und dem nationalsozialistischen System der Zwangsarbeit her, sondern waren eben auch ein Produkt der Deportationen (Kapitel 34). Die russischsprachige Historiographie geht von einem Absinken der Bevölkerung von gut 1,2 Millionen vor 1941 auf ca. 350.000 im Sommer 1944 aus. $^{20}$ Bei der auf die Deportationen folgenden Ansiedlung wurden, wie bereits erwähnt, russische und ukrainische Familien bevorzugt, die im landwirtschaftlichen Bereich arbeiteten, der neben dem Tourismus einen ausgeprägten Sektor in der Ökonomie der Halbinsel darstellte. Trotz diverser Rückschläge, etwa durch die Rücksiedlung unzufriedener NeusiedlerInnen, übertraf die Einwohnerzahl schon 1959 die der Vorkriegszeit um ca. 75.000. ${ }^{21}$ Dennoch blieb der Mangel an Arbeitskräften ein drängendes Problem. Es war nur eines von vielen, denn wirtschaftlich fiel die Krim auch wegen von der Zentrale zu verantwortender Investitionsdefizite und schlechter Planungen im Vergleich mit anderen Regionen der Ukrainischen SSR oder auch der RSFSR zurück. Sasse verweist in diesem Zusammenhang auf die Problematik sowjetischer Daten, welche eine Einschätzung der tatsächlichen Leistungsfähigkeit der Wirtschaft der Krim erschweren. Tatsache ist, dass die Meldungen über qualitative und quantitative Produktionssteigerungen häufig nur wenig mit der Realität korrelierten. Sie waren Teil der Propaganda, um die realsozialistische Welt (und am besten auch die außerhalb) von der Systemüberlegenheit der Sowjetunion zu überzeugen. Nur

17 Siegelbaum (2018); Qualls (2009), $109 \mathrm{f}$.

18 Qualls (2009), $1 \mathrm{f}$.

19 Vgl. Tymčenko (2014).

20 Djuličev (2006), $196 \mathrm{f}$.

21 Maksimenko (1990), 58. 
partiell ist feststellbar, woher die Mittel für den Wiederaufbau und die weitere Wirtschaftsförderung kamen - von Moskau oder von Kiew?22 Zur Beantwortung der Frage, inwieweit die Übergabe der Krim in ukrainische Hände ein Erfolg war, wäre dies aber wichtig. Sasse kommt letztlich zu dem Ergebnis, dass sich bei Großprojekten wie dem Nord-Krim-Kanal eine „hierarchy of decision making“ ausgebildet hatte, die von Moskau und dem allmächtigen staatlichen Wirtschaftsplanungskomitee „Gosplan“ (Gosudarstvennyj planovyj komitet) über Kiew und erst dann nach Simferopol' verlief. ${ }^{23}$

Eine gewisse eigene Handlungsmacht konnte das ab 1965 dann auch offiziell mit dem Titel „Heldenstadt“ geehrte Sevastopol' ausüben: Von 1948 bis in die 1990er Jahre hinein verfügte die wegen ihrer militärstrategischen Bedeutung selbst von SowjetbürgerInnen nur mit einer Sondergenehmigung zu besuchende geschlossene Stadt über ein eigenes, selbst zu verantwortendes Budget. ${ }^{24}$ Zudem besaß sie, neben Moskau und Leningrad, als dritte Stadt der Sowjetunion den Sonderstatus des „föderalen Ranges“. Ein populärer russischer Mythos besagt, dass die Stadt nicht nur wegen ihrer Verdienste während des Krimkriegs und des Zweiten Weltkriegs zu dieser Auszeichnung gekommen sei, sondern auch - eher banal - weil Stalin 1948 bei einem Besuch der Stadt feststellen musste, dass der Wiederaufbau nur schleppend vorangehe. Die Sonderverwaltung stellte daraufhin weitere finanzielle Mittel zur Verfügung. ${ }^{25}$ Der rechtliche Status Sevastopol's kann hier nur im Ansatz dargestellt werden, wichtig ist aber Folgendes: In den Debatten über die angenommene Unrechtmäßigkeit des Transfers der Krim 1954 in den Bestand der Ukrainischen SSR wird russischerseits zuweilen argumentiert, Sevastopol' sei als Stadt föderalen Ranges stets russisch und niemals ukrainisch gewesen (vgl. Kapitel 37).

Sevastopol' war als Kriegshafen gegründet worden und sollte somit primär den militärischen Erfordernissen der russischen bzw. später dann sowjetischen Flotte genügen. Dennoch waren an die Stadt von Anbeginn auch wirtschaftliche Erwartungen geknüpft worden, die sich jedoch über die Zeit nur eingeschränkt erfüllen sollten. ${ }^{26}$ Standortgemäß dominierten Schiffsbau, Fischerei, Weinproduktion sowie kleinere Textil- und Lebensmittelbetriebe die städtische Wirtschaft. Der Aufbau einer Großindustrie wurde durch den felsigen Untergrund in der Umgebung verhindert. ${ }^{27}$ Beim Wiederaufbau der zerstörten Stadt zeigten ihre

22 Sasse (2007), $122 \mathrm{f}$.

23 Sasse (2007), 124.

24 Vaneev (1983), 22.

25 Vgl. hierzu die Diskussion bei Hromenko (2017), 178-203.

26 Vgl. Jobst (2017b), $172 \mathrm{f}$.

27 Qualls (2009), 13. 
BewohnerInnen Handlungsmacht, vielleicht sogar einen kollektiven Eigensinn, der in der unmittelbaren Nachkriegszeit mit dem Wiederaufleben des Stalinismus durchaus heldenhafte Züge trug. Wie Karl Qualls zeigen konnte, verweigerten sich die lokalen Akteure beispielsweise der zeittypischen architektonischen Tendenz im Städtebau, welche als „Zuckerbäckerstil“ bezeichnet wird. Stattdessen wurde die Stadt mehr oder weniger nach ihrem historischen Vorbild rekonstruiert: Letztlich (und bis heute) vereint Sevastopol's Architektur einen primär russischen Charakter mit sowjetischen Zitaten - sie steht für „the re-creation of glorious past and future promise of the great Soviet utopia.“28

Dass eine so große Zahl ehemaliger SowjetbürgerInnen unabhängig ihrer ethnischen Herkunft so positive Gedanken mit der Krim verbindet, hängt nicht nur mit der populären Erzählung von der „Heldenstadt“ Sevastopol’ zusammen, sondern auch mit der schwunghaften Entwicklung der Halbinsel zum wohl wichtigsten Ort des sowjetischen Massentourismus. Die Krim hatte ja schon immer Reisende angelockt, wurde geradezu zu einem Sehnsuchtsort, aber erst in der zweiten Hälfte des 20. Jahrhunderts wurde sie eine Destination für die Volksmengen. ${ }^{29}$ Die Formen und die Kontexte des Reisens entwickelten eine große Bandbreite: Neben die bereits in zarischer Zeit begründete Sanatoriumskultur traten von Gewerkschaften und sonstigen gesellschaftlichen Organisationen durchgeführte Reisen (oftmals in eigene Heime und Unterkünfte) sowie der Individualtourismus, der auch in der Sowjetunion möglich war. Ein spezifisches Phänomen des Reisens kam der Verwirklichung der "great Soviet utopia“ wohl am nächsten: die sowjetische Pionierlagerkultur, deren unbestrittene ,Hauptstadt ${ }^{\star}$ das 1925 in der Nähe von Gurzuf gegründete „Artek“ wurde. ${ }^{30}$ Unter den zahlreichen Pionierlagern in der UdSSR und im sog. Ostblock war es das bekannteste, größte und prestigeträchtigste. Es besteht bis heute, wenngleich unter gewandelten Vorzeichen. ${ }^{31}$ „Artek“ wurde ein Synonym für die kollektiven Träume mehrerer Generationen junger Sowjetmenschen. Diese verwirklichten sich nicht für alle, denn nur diejenigen Kinder, die sich in Schule und Jugendorganisation besonders verdient gemacht hatten, durften ihre Ferien dort verbringen. So zumindest in der Theorie, denn praktisch gelangten auch die weniger begabten Sprösslinge der sowjetischen Nomenklatura in das Ferienlager. „Artek“ sollte genauso außerhalb der realsozialistischen Welt von der reklamierten systemischen Überlegenheit künden und wurde deshalb zugleich ein Zentrum des in-

28 Qualls (2009), 5.

29 Zur Entwicklung des sowjetischen Tourismus im Allgemeinen vgl. Koenker (2013).

30 Dazu als Einstieg Furin u. Rybinski (1975).

31 Vgl. die Website Artek (2018), die gegenwärtig das Lager mit dem Motto „In der Sonnenstadt“ [V solnečnom gorode] bewirbt. 


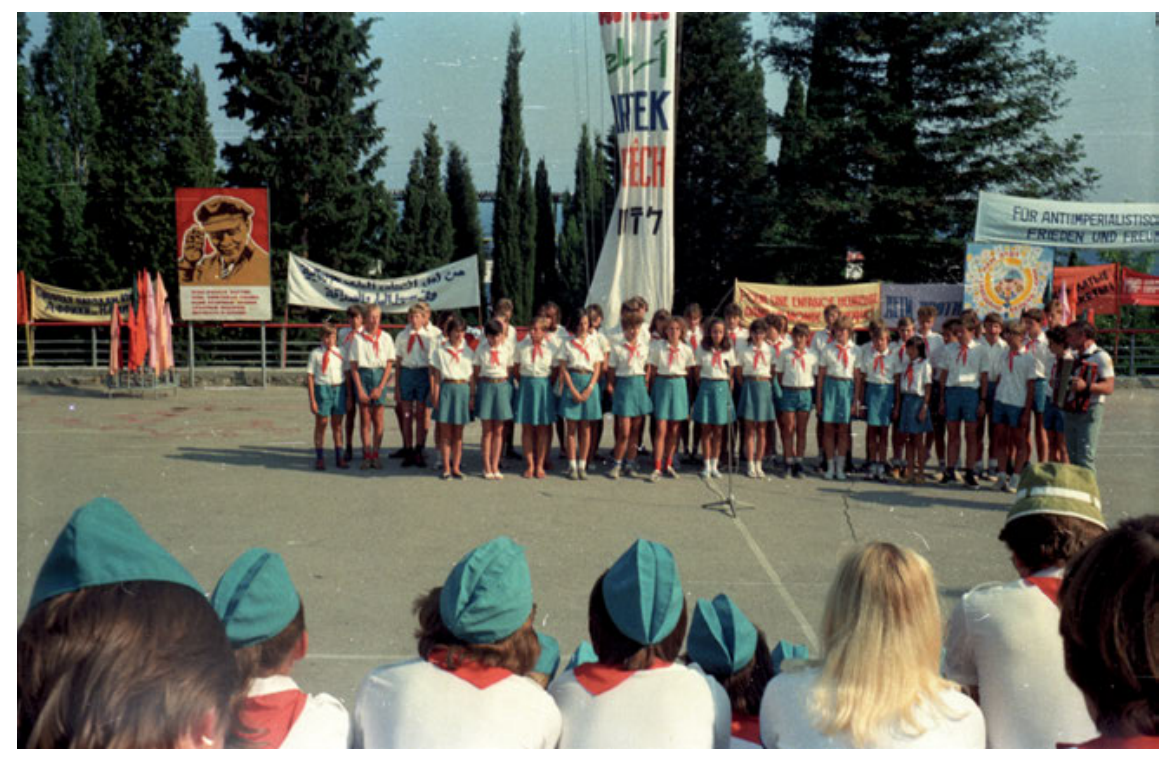

Abb. 14: Pionierlager Artek, 1986

ternationalen Kinder- und Jugendaustausches. Die Pionierlager waren Teil einer möglichst früh, also im Kinder- und Jugendalter greifenden und der Kleinfamilie entzogenen Erziehung zum sog. Neuen (= sozialistischen) Menschen. Die ideologische Indoktrination durch Erziehung war zum Teil durchaus kindgerecht ${ }^{32}$, junge Talente wurden gesucht und gefördert, berühmte Persönlichkeiten wie der Kosmonaut Jurij Gagarin (1934-1968) oder in- und ausländische Staatschefs wie Walter Ulbricht (DDR, 1893-1973), Georgi Dimitrov (Bulgarien, 1882-1949) oder Ho Chi Minh (Vietnam, 1890-1969) besuchten „Artek“. Die weitläufige, aus mehreren Haupt- und Nebenlagern bestehende Anlage setzte auch architektonisch Maßstäbe, besonders der Ausbau zwischen 1957 und 1966, der zudem die Kapazitäten so erhöhte, dass ab Mitte der 1960er Jahre jährlich über 20.000 Kinder und Jugendliche gleichzeitig dort Ferien machen konnten. ${ }^{33}$

Insgesamt war die wirtschaftliche Entwicklung der Halbinsel nach dem Zweiten Weltkrieg dennoch keine Erfolgsgeschichte, trotz des wichtigen Fremdenverkehrssektors, der Lebensmittelindustrie (z. B. Fischkonservierung) oder vereinzelter industrieller Großanlagen wie das Eisenerz-Kombinat in Kerč'

32 Vgl. Kelly (2007), 548f.

33 Winkelmann (2003). 
(„Kamyš-Burunskij-Eisenerz-Kombinat“). Dies gilt letztlich auch für den traditionell bedeutenden Agrarbereich. Neben dem Tabakanbau ${ }^{34}$ sind hier vor allen Dingen Obst und Gemüse sowie Wein zu nennen; dies waren Bereiche, die immer wieder von Rückschlägen heimgesucht wurden, die allerdings weniger der ukrainischen Verwaltung anzulasten waren, sondern vielmehr im gesamtsowjetischen Kontext zu verstehen sind..$^{35}$ Außerhalb des sog. Ostblocks machte die Wirtschaft der Halbinsel einmal besonders von sich reden: in der Mitte der 1980er Jahre durch die sog. Antialkohol-Kampagne des letzten Generalsekretärs der KPdSU, Michail Gorbačev ( $\left.{ }^{\star} 1931\right)$. Diese war nur eine von diversen Initiativen gegen den übermäßigen Genuss alkoholischer Getränke in sowjetischer Zeit; die der Jahre 1985 bis 1987 erregte aber das größte Aufsehen. Abstinenz sollte die Produktivität und die Lebenserwartung vor allen Dingen der Männer in der UdSSR steigern und die Kriminalität senken. Die unter dem Motto „Nüchternheit ist die Lebensnorm“ (russ.: „Trezvost' - Norma žizni“) durchgeführte Kampagne bestand aus einem ganzen Bündel von Maßnahmen, u. a. der Verteuerung des Produkts, Einschränkung der Verkaufsstellen und Öffnungszeiten, aber eben auch der drastischen Reduzierung von Anbauflächen. Für die Krim mit ihren im 19. Jahrhundert zu Weltruhm gelangten Weingütern wie Massandra bedeutete dies nichts anderes als eine Katastrophe, wurden doch alte und wertvolle Rebsorten zum Teil für immer zerstört. ${ }^{36}$

Die Betrachtung der Geschichte der Halbinsel Krim in der Nachkriegszeit wäre nicht vollständig ohne einen Hinweis auf das Schicksal der deportierten KrimtatarInnen, die seit 1944 mehrheitlich in Zentralasien leben mussten. Auch fern der Heimat hatten die meisten von ihnen die Krim nicht vergessen, sondern bewahrten generationenübergreifend eine lebhafte Erinnerung an diese, wie die Ethnologin Greta Lynn Uehling hat zeigen können. ${ }^{37}$ Krimtatarische AkteurInnen beließen es in sowjetischer Zeit trotz aller Pressionen nicht dabei, nur von der „Rückkehr“ (krimtat.: avdet) auf die Halbinsel zu träumen, sondern versuchten diese unter der Ausnutzung weitgehend legaler Möglichkeiten aktiv voranzutreiben. Wie so viele SowjetbürgerInnen, so hatte auch die krimtatarische Bevölkerung nach Stalins Tod auf Erleichterungen ihres harten Schicksals gehofft. Sie wurden insofern enttäuscht, als dass diese Nationalität - wie dargelegt - in der sog. Geheimrede Chruščevs von 1956 eben nicht genannt und erst zwei Dekaden später rehabilitiert wurde. In den sog. Spezialsiedlungen, in denen krim-

34 Auch deutsche Unternehmen waren während der Besatzungszeit unter brutaler Ausnutzung örtlicher Arbeitskräfte im Bereich der Tabakproduktion tätig: Roth u. Abraham (2011).

35 Vgl. Sasse (2007), 123.

36 Zur Bewertung der Folgen der Kampagne vgl. Latyš (2010).

37 Uehling (2004). 
tatarische und andere deportierte Nationalitäten untergebracht waren, kam es im Verlauf der 1950er Jahre immerhin zu besseren Lebensbedingungen. Vordem jedoch war die Todesrate hoch, selbst wenn man die offiziellen sowjetischen Zahlen zugrunde legt: Nach diesen sollen innerhalb von achtzehn Monaten nach der Deportation im Frühjahr 1944 ca. achtzehn Prozent der krimtatarischen Bevölkerung gestorben sein; krimtatarische Quellen gehen - wenig erstaunlich - mit 46 Prozent von einer deutlich höheren Zahl aus. ${ }^{38}$ Signifikant bei ethnischen Säuberungen und auch im krimtatarischen Fall ist die relativ höhere Opferzahl unter Kindern und Frauen. ${ }^{39}$ In den Nachkriegsjahren versuchte die krimtatarische Diaspora mit unterschiedlichem Erfolg, sich in die neue Umgebung einzuleben und mit der lokalen Bevölkerung zu arrangieren. Dies gelang, wie Williams auf der Grundlage von Interviews hat feststellen können, mal besser und mal schlechter: Während in Kasachstan das Verhältnis zwischen Neu- und AltsiedlerInnen in der Regel auskömmlich war, traf dies in der Republik Mari oder im Hauptdeportationsgebiet der Usbekischen SSR weniger $\mathrm{zu}^{40}$

Eine wirkliche Zäsur in der krimtatarischen Frage bedeutete das Jahr 1967, in der es zu einer Teilrehabilitierung der KrimtatarInnen durch den Obersten Sowjet der UdSSR kam. Sie wurden von dem Vorwurf der Massenkollaboration freigesprochen, aber immer noch blieb ihnen das Recht auf Rückkehr auf die Krim versagt. Interessant war die gewählte Sprachregelung: Bezeichnenderweise richtete sich das Dekret nämlich an die „tatarische Nationalität, die früher die Krim bewohnte.“ Das Wort „krimtatarisch“ wurde vermieden und nichts deutete daraufhin, dass die „tatarische Nationalität“ dereinst wieder die Halbinsel bewohnen würde. Zudem wurde das die KrimtatarInnen entlastende Schriftstück nicht in der ganzen Sowjetunion veröffentlicht, sondern allein in den Deportationsgebieten. ${ }^{41}$ Außerhalb dieser blieb diese Nationalität somit für viele SowjetbürgerInnen weiterhin ein Volk von Verrätern. Vereinzelte krimtatarische Familien, die sich nach 1967 auf eigene Faust auf die Krim zurückschlugen, wurden von den örtlichen Behörden zumeist zurückgewiesen. ${ }^{42}$

In der Auseinandersetzung über das Erbe und die Folgen des Stalinismus mit seinen großen Opferzahlen war seit den späten 1950er Jahren eine DissidentInnenbewegung entstanden. Durch die Vervielfältigung und Verbreitung von Schriften im Selbstverlag (russ.: Samizdat) wurde die staatliche Zensur umgangen und es bildete sich eine wenn auch kleine Gegenöffentlichkeit heraus, die anti-

38 Marples u. Duke (1995), 277.

39 Naimark (2008), 132.

40 Williams (2001), 391.

41 Vgl. den vollständigen Text bei Fisher (1978), 179.

42 Naimark (2008), 133. 
stalinistisch, aber insgesamt systemimmanent argumentierte. Seit Mitte der 1960er Jahre, vor allen Dingen aber in dem global so entscheidenden Jahr 1968 traten die DissidentInnen zunehmend in das Licht der Öffentlichkeit. Auch krimtatarische Akteure als Angehörige einer besonders von den stalinistischen Repressionen betroffenen Gruppe spielten in dieser Bewegung eine Rolle. Einer von ihnen und bis heute die wichtigste und bekannteste Gallionsfigur der krimtatarischen Bewegung ist Mustafa Cemilev (krimtat.; ukr.: Mustafa Džemiljev; russ.: Mustafa Džemilev). Der 1943 in der Nähe von Sudak geborene spätere Aktivist wurde im Kleinkindalter mit seinen Eltern nach Zentralasien deportiert. Seit Ende der 1950er Jahre engagierte er sich mit Petitionen für die krimtatarische Sache und geriet deshalb mit der Sowjetmacht in Konflikt. Beruflich legte man ihm immer wieder Steine in den Weg, so durfte er beispielsweise nicht wie gewünscht Orientalistik studieren. Wegen sog. anti-sowjetischer Aktivitäten 1966 erstmalig verhaftet, saß er insgesamt fünfzehn Jahre in sowjetischen Gefängnissen bzw. Lagern ein, was ihm einen „Mandela-ähnlichen“ Status einbrachte. Er trat in der Gefangenschaft wiederholt in mehrmonatige Hungerstreiks, die er nur wegen der von der Gefängnisleitung angeordneten Zwangsernährung überlebte. ${ }^{43}$ Die sowjetischen Behörden fürchteten ganz offenbar die Verehrung Cemilevs als Märtyrer, der dieser für seine Landsleute wurde. 1969 war er gemeinsam mit anderen sowjetischen Aktivisten wie Viktor Krasin (1929-2017) Gründungsmitglied der „Initiativgruppe zur Verteidigung der Menschenrechte in der Sowjetunion“, die u.a. internationale Organisationen mit Informationen zu Menschenrechtsverletzungen in der Sowjetunion versorgten. ${ }^{44}$ Cemilev wurde neben Andrej Sacharov (1921-1989) und dem ehemaligen hohen Militär Petr Grigorenko (russ.; ukr.: Petro Hryhorenko; 1907-1987) eine der populärsten Figuren der DissidentInnenszene. An der Verbindung zwischen Grigorenko und der krimtatarischen Bewegung ist zu zeigen, dass das Schicksal des sürgün - also der Deportation von 1944 - auch außerhalb tatarischer oder muslimischer Diskursgruppen als Unrecht empfunden wurde. Dieses wurde auch beim Namen genannt: In einer bis heute als legendär geltenden Rede in Moskau vor für ihre Rückkehr auf die Krim protestierenden KrimtatarInnen 1968 erklärte Grigorenko, das spätere Gründungsmitglied der Moskauer Helsinki-Gruppe für Menschenrechte und zeitweise ihr inoffizieller Vorsitzender, deren Deportation für verfassungswidrig. Und er forderte sie auf, nicht aufzugeben: „So begin to demand. And demand not just parts, pieces, but all that was taken from you unlawfully - demand the reestablishment

43 Zur Biographie Cemilevs vgl. u.a. Williams (2001), 427-430, Zitat 427.

44 Daniel A. (2016). 
of the Crimean Autonomous Soviet Socialist Republic! [Stormy applause and cries of ,Hail the Crimean Autonomous Soviet Socialist Republic '].“445

Die Reaktion des anwesenden krimtatarischen Publikums auf die Rede des später in die USA emigrierten und dort auch verstorbenen Grigorenkos - „Hail the Crimean Autonomous Soviet Socialist Republic“ - weist darauf hin, dass die meisten DissidentInnen einschließlich der krimtatarischen lange innerhalb des Systems standen und agierten. Im krimtatarischen Fall war der politische Bezugspunkt bis in die Zeit Gorbačevs nicht die Auflösung der UdSSR, die staatliche Unabhängigkeit oder die Wiederauferstehung eines Krim-Chanats mit mehr oder weniger engen Verbindungen zur Türkei, sondern die Wiedererrichtung der ASSR. Die sowjetische Führung wusste aber im Fall der Krim diesen Umstand nicht zu nutzten. Die seit dem Beginn der 1980er Jahre von sowjetischen Behörden begonnenen Überlegungen zur Schaffung einer tatarischen Ersatz-Heimat weitab von der Nordküste des Schwarzen Meeres in den trockenen Steppengebieten südlich von Samarkand und Buchara stießen bei den zukünftigen BewohnerInnen auf Protest. ${ }^{46}$ Die enge emotionale Bindung der krimtatarischen Bevölkerung zur Halbinsel verstanden die zumeist russischstämmigen Vertreter der sowjetischen Nomenklatura offenbar nicht. Und dies obwohl gerade die russische Bevölkerung selbst eine so enge Beziehung zur Halbinsel entwickelt hatte, dass sie diese für ein unveräußerliches russisches Terrain hielt und von „unserer Krim“ (russ.: Krym naš) sprach. Es zeigt aber auch, wie wenig sich die politischen Eliten von der bereits in der frühen Sowjetunion entwickelten Strategie der nationalen Territorialisierung gelöst hatten, nach der ein jeder Ethnos ,sein` Territorium erhalten sollte. Für die KrimtatarInnen stand aber fest, dass ihr Territorium allein die Krim war. Das Ende der Sowjetunion brachte sie diesem Ziel näher.

Die schon zu anderen Zeiten für die Krim als imperiale Peripherie geltende Regel, dass die Ereignisse im Zentrum zumeist einen (manchmal abgeschwächten) Widerhall auf der Halbinsel zeitigten, wiederholte sich am Ende der Sowjetunion abermals, wobei Zeitgenossen freilich nicht klar gewesen sein dürfte, dass sie in einem sich auflösenden Imperium lebten. Weniger der von Gorbačev verordnete „Umbau“ (die sog. Perestrojka) als vielmehr die gleichfalls von ihm gewünschte „Transparenz“ (also die Glasnost') eröffnete den SowjetbürgerInnen einen größeren Kommunikationsraum als bisher. Für die in der Diaspora lebenden KrimtatarInnen führten die weggefallenen Sprechverbote $\mathrm{zu}$ einer massiven Mobilisierung. Doch auch auf der überwiegend slavischen Krim selbst, veränderte sich die Lage. Die Reaktorkatastrophe von Čornobyl' im April 1986 hatte vor allem

45 Die vollständige Rede in englischer Übersetzung vgl. bei Grigorenko (2018).

46 Dazu Williams (2001), 430-433. 
in der Ukraine zur Formierung zivilgesellschaftlicher Kräfte geführt. Es verschränkten sich nationale und umweltpolitische Forderungen, wobei letztere schnell zu Gunsten nationaler Postulate an Gewicht verloren. ${ }^{47}$ Auch auf der Krim formierte sich eine ,grüne‘ Bewegung, die sich vor allen Dingen gegen die Moskauer Pläne zur Errichtung eines Atomkraftwerkes auf der Halbinsel richtete. Sogar die örtliche Kommunistische Partei machte sich diesen Standpunkt zu eigen und verfügte im Frühjahr 1989 ein Moratorium - und zwar ohne Rücksprache mit den eigentlich zuständigen Stellen in Moskau; Kiew war dafür ohnehin nicht zuständig. ${ }^{48}$

Die Parteieliten der Halbinsel setzten sich seit Mitte der 1980er Jahre ohnehin vom offiziellen Moskauer Kurs ab. Dies geschah allerdings nicht, weil ihnen Gorbačevs Pläne zum Umbau des sowjetischen Systems nicht weit genug gingen, sondern weil sie ihnen zu weit gingen; die russophone Nomenklatura war nämlich - und blieb es bis heute - stark strukturkonservativ, diffus an sowjetischen gesellschaftlichen und politischen Vorstellungen verhaftet. Dies lag nicht zuletzt daran, dass die schöne Krim ein bevorzugter Ort für pensionierte, aber noch immer gut vernetzte Funktionsträger geworden war. So nimmt es nicht wunder, dass bereits Ende der 1980er Jahre aus diesem Kreis Pläne laut wurden, welche an die vermeintlich guten Jahre der frühen Sowjetunion erinnerten, an die vorstalinistische Zeit nämlich: Der Krim sollte danach der Vorkriegsstatus einer Autonomen Sozialistischen Sowjetrepublik (ASSR) gegeben werden, u.U. als wirtschaftliches Sondergebiet innerhalb der gerade noch existierenden Sowjetunion. Zugleich hoffte man so, den stärker werdenden tatarischen Zustrom kanalisieren zu können. In pragmatischer Ausnutzung der ansonsten abgelehnten GorbačevPolitik wurde im Jänner 1991 ein Referendum zur Re-Installierung einer ASSR der Krim durchgeführt. Erstaunliche 93 Prozent der an der Abstimmung teilnehmenden örtlichen Bevölkerung sprachen sich dafür aus. Die Enttäuschung war allerdings groß, als der formal ja für die Krim zuständige Oberste Sowjet der Ukraine die „Wiederbegründung der ASSR Krim“ nur im Bestand der Ukrainischen SSR annahm. ${ }^{49}$ Selbstverständlich hatte die Mehrheit der Krimbevölkerung auf die Anbindung an die russländische Sowjetrepublik gehofft. Sowohl in der sowjetischen Verfassung von 1977 als auch der der Ukrainischen SSR war so ein autonomes Gebilde zwar nicht vorgesehen, in der insgesamt recht unübersichtlichen Zeit am Ende der UdSSR wurde der ASSR-Plan jedoch von Kiew adaptiert. ${ }^{50}$

47 Für die Ukraine ohne die Halbinsel vgl. z.B. Jobst (2011c). Zum sowjetischen Phänomen des sog. Ökonationalismus vgl. Dawson (1996).

48 Sasse (2007), $131 \mathrm{f}$.

49 Im Detail dazu Sasse (2007), 133-140.

50 Vgl. Magocsi (2014), $134 \mathrm{f}$. 
In den folgenden Jahren führte dies zu Problemen zwischen Simferopol', dem alten und neuen politischen Zentrum der Halbinsel, und Kiew sowie zwischen der Ukraine und der Russländischen Föderation.

Es ist für die eigentümliche prorussische/prosowjetische Gemengelage selbst unter der ukrainischstämmigen Bevölkerung auf der Krim bezeichnend, dass sich die in der Nach-Čornobyl'-Zeit ab 1986 so lebhaft ausbildenden ukrainischen zivilgesellschaftlichen Bewegungen dort nicht oder nur kurzfristig bemerkbar machten. Von der schnell von alten kommunistischen Kadern ,gekaperten‘ AntiAtomkraft-Bewegung auf der Krim abgesehen, waren dissidente Gruppen wie „Narodnyj Ruch Ukraïny“ („Nationale Bewegung der Ukraine“; kurz: Ruch), die in der Ukraine selbst eine große Rolle spielten, nur vorübergehend sichtbar. ${ }^{51}$ Und auch die in den 1970er Jahren in der ganzen Sowjetunion ihre Spuren hinterlassende sog. Helsinki-Bewegung hatte auf der Krim selbst unter der slavischen Bevölkerung kaum Widerhall gehabt; anders als unter KrimtatarInnen, welche sich jedoch zu jenem Zeitpunkt überwiegend noch außerhalb der Halbinsel aufhielten. ${ }^{52}$

51 Über den Anteil des „Ruch“ beim Auflösungsprozess der UdSSR vgl. Haran u. Prokoptschuk (2013).

52 Saal (2014); Peter u. Wentker (2012). 
